\title{
Power Dissipation of Memristor-Based Relaxation Oscillators
}

\author{
Mohammed E. FOUDA ${ }^{1}$, Ahmed G. RADWAN $N^{1,2}$ \\ ${ }^{1}$ Dept. of Engineering Mathematics, Faculty of Engineering, Cairo University, 12613 Giza, Egypt \\ ${ }^{2}$ Nano-electronic Integrated Systems Center (NISC), Nile University, Giza, Egypt \\ m_elneanaei@ieee.org, agradwan@ieee.org
}

\begin{abstract}
Recently, many reactance-less memristive relaxation oscillators were introduced, where the charging and discharging processes depend on memristors. In this paper, we investigate the power dissipation in different memristor based relaxation oscillators. General expressions for these memristive circuits as well as the power dissipation formulas for three different topologies are derived analytically. In addition, general expressions for the maximum and minimum power dissipation are calculated. Finally, the calculated expressions are verified using PSPICE simulations showing very good matching.
\end{abstract}

\section{Keywords}

Memristor, power, reactance-less, memristive circuits, oscillators

\section{Introduction}

Recently, memristors have proved their promising abilities in different applications. The history of the memristor dates back to 1971 when Chua postulated the existence of the missing element [1]. Then later, in 2008, HP announced that they found the missing element and presented the first simple model of the memristor [2]. Since this date, the memristor has received intensive attention from the research community in various fields. Different models have been introduced depending on the physical implementation of the memristor [3], however, the solid state samples are not commercially available yet. So many emulator circuits are designed to mimic the behavior of the memristor [5], [6], [7], [8]. In addition, different SPICE models were presented to fit the memristor behavior [9], [10], [11]. During the last few years, several papers have been published about the memristor and its unique characteristics besides its applications. Memristors are used in chaotic systems, non-volatile memory, pattern recognition, neural networks, digital circuits, programmable analog circuits, circuit modeling, and oscillators [3], [4].

Oscillators are one of the basic building blocks of any electronic systems. Recently, the memristor has been used to build the oscillator circuits - for instance, the memristor has been used instead of resistors in sinusoidal oscillators such as Wien oscillators [12]. In the memristor based Wien oscillator, the poles of the system oscillate however sustained oscillation is maintained owing to the properties of the memristor. Recently, reactance-less memristive relaxation oscillators are a novel topic in the circuit theory, where the charging and the discharging of the reactive elements is emulated by the memristor. Different realizations of symmetric and asymmetric memristor based relaxation oscillators have been introduced in [13], [14], [15]. The main advantage of the memristor-based oscillators is that they are fully integrable on-chip due to their nano-scale dimensions. However, in case of the low frequency relaxation oscillator, a large value capacitor is needed which is not integrable on-chip.

In [2], the current-voltage relationship of the memristor was described by

$$
\begin{gathered}
v(t)=\left(x R_{\mathrm{on}}+(1-x) R_{\mathrm{off}}\right) i(t), \\
\frac{d x}{d t}=k i(t)
\end{gathered}
$$

where $R_{\text {on }}$ and $R_{\text {off }}$ are the minimum and maximum achievable resistances of the memristor respectively, $k=\left(\mu_{\mathrm{v}} R_{\mathrm{on}} / D^{2}\right) \Omega / C$, and $\mu_{\mathrm{v}}$ is the ion mobility of the memristor.

This paper is organized as follows: Section 2 discusses the memristor-based reactance-less oscillators. In Section 3, the power consumption in the memristive circuit is discussed to be used in calculating the power of the relaxation oscillator. Then, the power dissipations in the memristor-based oscillators are calculated for different cases and verified with the PSPICE simulations in Section 4. Finally, the conclusion is given.

\section{Memristor-Based Reactance-less Oscillators}

The memristor based relaxation oscillator consists of a logic circuit and a memristive circuit as shown in Fig. 1. The memristive circuit consists of a memristor in series with either resistor or memristor as discussed in [13], [14], [15]. Thus, the power consumption in the oscillator circuit is mainly from the memristive circuit. So, it is essential to investigate the power consumption in the memrstive circuit. 
The analysis of the memristive circuit was previously discussed in [15] where four different cases were defined. These cases are shown in Fig. 2. It is worth noting that when $k=0$, the memristor equation tends to constant "resistor". The memristance $R_{b}$ is either constant or given by

$$
R_{\mathrm{b}}(t)=\frac{-R_{\text {ind }} \pm \sqrt{\left(R_{\mathrm{ind}}+(\alpha+1) R_{\mathrm{inb}}\right)^{2}-2(\alpha+1) k_{\mathrm{b}}^{\prime} \varphi(t)}}{\alpha+1},
$$

where $R_{\text {ind }}=R_{\text {ina }}-\alpha R_{\text {inb }}, R_{\text {ina }}$ and $R_{\text {inb }}$ are the initial memristances of $R_{\mathrm{a}}$ and $R_{\mathrm{b}}$ respectively, $k^{\prime}=k\left(R_{\mathrm{off}}-R_{\mathrm{on}}\right)$ and $\alpha=k_{\mathrm{a}}^{\prime} / k_{\mathrm{b}}^{\prime}$ representing memristors mismatch. Also, the memristance $R_{\mathrm{a}}$ is either constant or given by

$$
R_{\mathrm{a}}(t)=\alpha R_{\mathrm{b}}(t)+R_{\text {ind }} .
$$

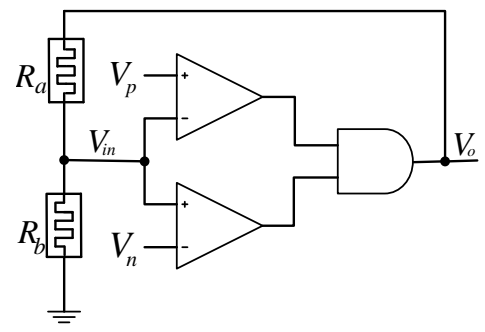

(a)

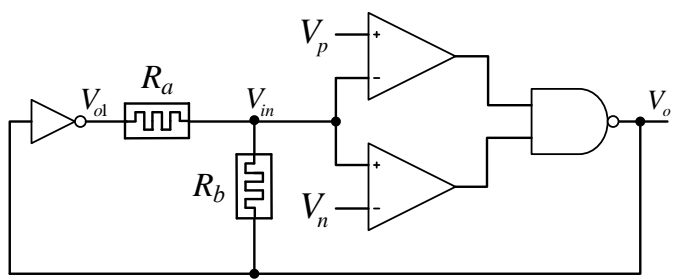

(b)

Fig. 1. Two generic architectures of a memristor-based oscillator, a) single gate oscillator and b) two gate oscillator.

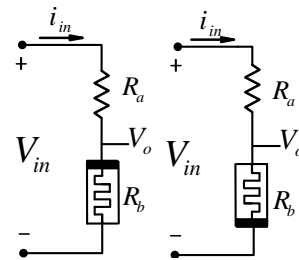

(a)

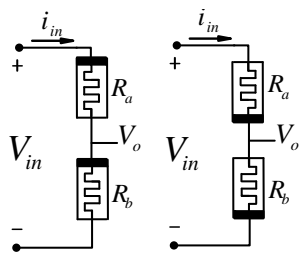

(c)

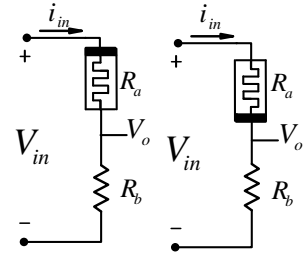

(b)

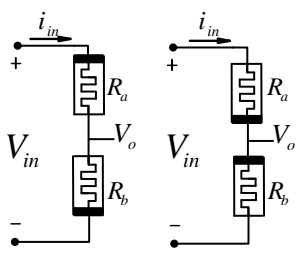

(d)
Fig. 2. Different cases of a memristive circuit, a) $\alpha=0$, b) $\alpha= \pm \infty$, c) $\alpha=1$, and d) $\alpha=-1$.

In the next section, the power consumption in the memristive circuit is discussed to be used in calculating the power of the relaxation oscillator.

\section{Power Consumption in the Memristive Circuit}

It is well known that the power consumption in two series resistors is given by

$p(t)=\frac{V_{\mathrm{in}}^{2}}{R_{\mathrm{a}}+R_{\mathrm{b}}}=\frac{V_{\mathrm{in}}^{2}}{\sqrt{\left(R_{\mathrm{ind}}^{2}+(\alpha+1) R_{\mathrm{inb}}\right)^{2}-2(\alpha+1) k_{\mathrm{b}}^{\prime} \varphi(t)}}$

and the power consumptions in the memristances $R_{\mathrm{a}}$ and $R_{\mathrm{b}}$ are given by

$$
p_{\mathrm{a}}(t)=\frac{V_{\mathrm{in}}^{2} R_{\mathrm{a}}}{\left(R_{\mathrm{a}}+R_{\mathrm{b}}\right)^{2}}, \quad p_{\mathrm{b}}(t)=\frac{V_{\mathrm{in}}^{2} R_{\mathrm{b}}}{\left(R_{\mathrm{a}}+R_{\mathrm{b}}\right)^{2}} .
$$

So the power dissipation in the four aforementioned special cases can be studied as follows:

1. $k_{\mathrm{a}}^{\prime}=0(\alpha=0)$, where memristance $R_{b}$ is reduced to

$$
R_{\mathrm{b}}(t)=-R_{\mathrm{a}}+\sqrt{\left(R_{\mathrm{a}}+R_{\mathrm{inb}}\right)^{2}-2 k_{\mathrm{b}}^{\prime} \varphi(t)}
$$

by substituting into (4) and (5), the power dissipation by $R_{\mathrm{a}}$ and the total power consumption are given by

$$
\begin{gathered}
p_{\mathrm{a}}(t)=\frac{V_{\mathrm{in}}^{2} R_{\mathrm{a}}}{\left(R_{\mathrm{a}}+R_{\mathrm{inb}}\right)^{2}-2 k_{\mathrm{b}}^{\prime} \varphi(t)}, \\
p(t)=\frac{V_{\mathrm{in}}^{2}}{\sqrt{\left(R_{\mathrm{a}}+R_{\mathrm{inb}}\right)^{2}-2 k_{\mathrm{b}}^{\prime} \varphi(t)}} .
\end{gathered}
$$

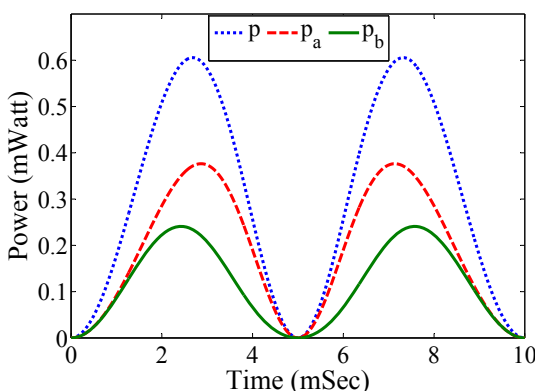

(a)

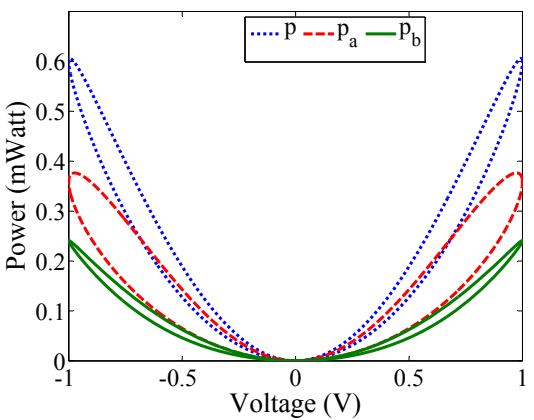

(b)

Fig. 3. Plot of power consumption in $R_{a}$ and $R_{b}$ for $\alpha=0$.

Figure 3(a) shows a plot of instantaneous power consumption of sinusoidal input $v(t)=\sin (2 \pi f t)$ for 
$R_{\mathrm{a}}=R_{\mathrm{inb}}=1 \mathrm{k} \Omega, k_{\mathrm{b}}^{\prime}=379 \mathrm{M} \Omega^{2} / \mathrm{C}$, and $f=100 \mathrm{~Hz}$. Figure 3(b) shows the power consumption versus applied voltage where a pinched hysteresis exists even for the power consumption in the resistor $R_{\mathrm{a}}$.

2. $k_{\mathrm{b}}^{\prime}=0(\alpha= \pm \infty)$, where the memristance $R_{a}$ reveals to

$$
R_{\mathrm{a}}(t)=-R_{\mathrm{b}}+\sqrt{\left(R_{\mathrm{b}}+R_{\mathrm{ina}}\right)^{2}-2 k_{\mathrm{a}}^{\prime} \varphi(t)},
$$

by substituting into (4), the total power consumption is

$$
p(t)=\frac{V_{\mathrm{in}}^{2}}{\sqrt{\left(R_{\mathrm{b}}+R_{\mathrm{ina}}\right)^{2}-2 k_{\mathrm{a}}^{\prime} \varphi(t)}} .
$$

Figure 4(a) shows a plot of power consumption for $R_{\mathrm{a}}=R_{\mathrm{inb}}=1 \mathrm{k} \Omega, k_{\mathrm{a}}^{\prime}=-379 \mathrm{M}^{2} / \mathrm{C}$, and $f=$ $100 \mathrm{~Hz}$. Figure 4(b) shows the power consumption versus applied voltage curve which demonstrates evensymmetric pinched hysteresis for power consumption in $R_{\mathrm{a}}, R_{\mathrm{b}}$ and the total power.

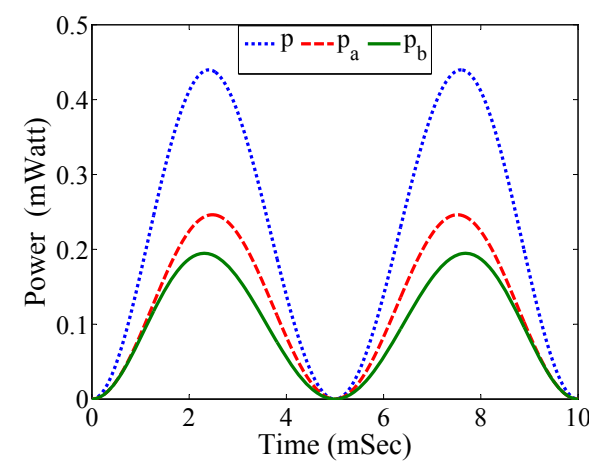

(a)

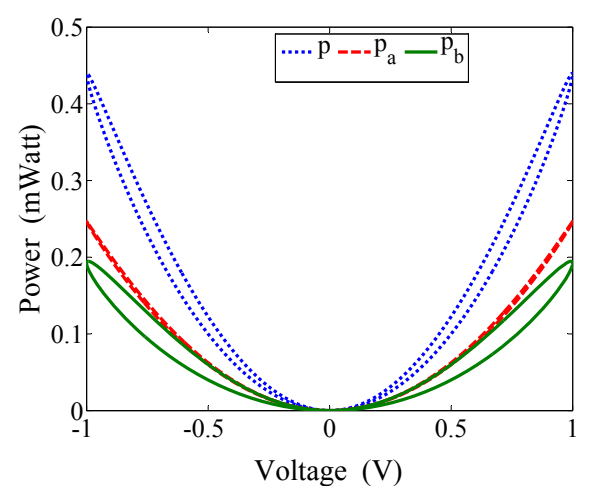

(b)

Fig. 4. Plot of power consumption in $R_{\mathrm{a}}$ and $R_{\mathrm{b}}$ for $\alpha= \pm \infty$.

3. $k_{\mathrm{a}}^{\prime}=k_{\mathrm{b}}^{\prime}=k_{\mathrm{ab}}^{\prime}(\alpha=1)$, the memristances $R_{\mathrm{a}}$ and $R_{\mathrm{b}}$ are

$$
\begin{aligned}
& R_{\mathrm{a}}(t)=\frac{R_{\mathrm{ina}}-R_{\mathrm{inb}}+\sqrt{\left(R_{\mathrm{inb}}+R_{\mathrm{ina}}\right)^{2}-4 k_{\mathrm{ab}}^{\prime} \varphi(t)}}{2}, \\
& R_{\mathrm{b}}(t)=\frac{R_{\mathrm{inb}}-R_{\mathrm{ina}}+\sqrt{\left(R_{\mathrm{inb}}+R_{\mathrm{ina}}\right)^{2}-4 k_{\mathrm{ab}}^{\prime} \varphi(t)}}{2},
\end{aligned}
$$

and the total power consumption is

$$
p(t)=\frac{V_{\mathrm{in}}^{2}}{\sqrt{\left(R_{\mathrm{inb}}+R_{\mathrm{ina}}\right)^{2}-4 k_{\mathrm{ab}}^{\prime} \varphi(t)}} .
$$

Figure 5(a) shows a plot of instantaneous power consumption due sinusoidal voltage excitation where $R_{\text {inb }}=$ $2 R_{\text {ina }}=2 \mathrm{k} \Omega, k_{\mathrm{ab}}^{\prime}=-379 \mathrm{M} \Omega^{2} \mathrm{~V}^{-1} \mathrm{~s}^{-1}$, and $f=$ $100 \mathrm{~Hz}$. Figure 5(b) shows the power consumptions versus the applied voltage where the power consumption in each memristor is pinched as well as the total power.

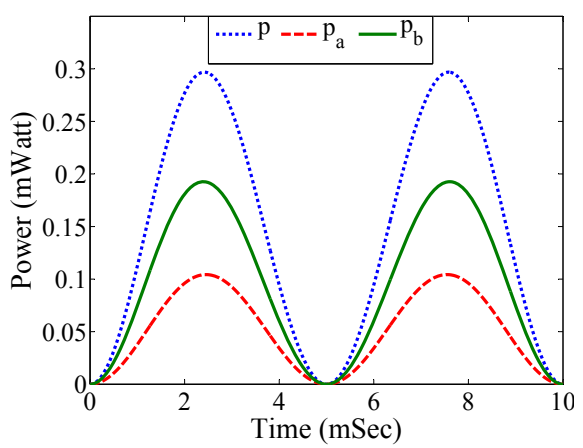

(a)

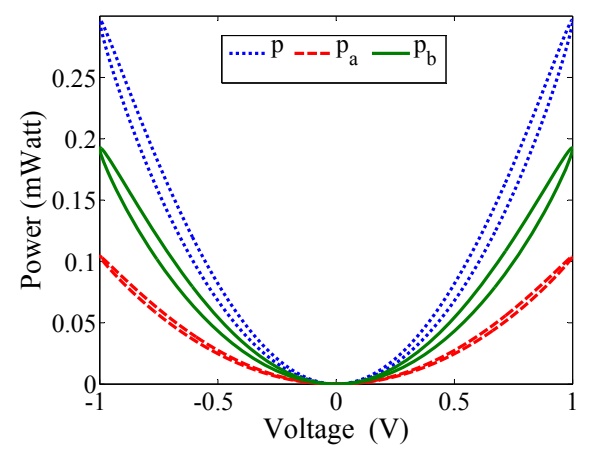

(b)

Fig. 5. Plot of power consumption in $R_{\mathrm{a}}$ and $R_{\mathrm{b}}$ for $\alpha=1$.

4. $k_{\mathrm{a}}^{\prime}=-k_{\mathrm{b}}^{\prime}(\alpha=-1)$, where the memristances $R_{\mathrm{a}}$ and $R_{\mathrm{b}}$ are

$$
\begin{aligned}
& R_{\mathrm{a}}(t)=R_{\text {ina }}-\frac{k_{\mathrm{a}}^{\prime}}{R_{\text {ina }}+R_{\text {inb }}} \varphi(t), \\
& R_{\mathrm{b}}(t)=R_{\text {inb }}-\frac{k_{\mathrm{b}}^{\prime}}{R_{\text {ina }}+R_{\text {inb }}} \varphi(t) .
\end{aligned}
$$

Thus, the total power consumption is

$$
p(t)=\frac{V_{\mathrm{in}}^{2}}{R_{\mathrm{ina}}+R_{\mathrm{inb}}} .
$$

Figure 6(a) shows a plot of instantaneous power consumption for $R_{\text {ina }}=R_{\text {inb }}=1 \mathrm{k} \Omega, k_{\mathrm{a}}^{\prime}=-k_{\mathrm{b}}^{\prime}=$ $-379 \mathrm{M} \Omega^{2} / \mathrm{C}$, and $f=100 \mathrm{~Hz}$. It is worth noting that the power consumption is pinched for each memristor, but the total power consumption is not pinched. Since, the power consumption in the anti connected 
memristances is proportional to the square of the applied voltage. However, in the other three cases, the power consumption has nonlinear pinched relation with the applied voltage.

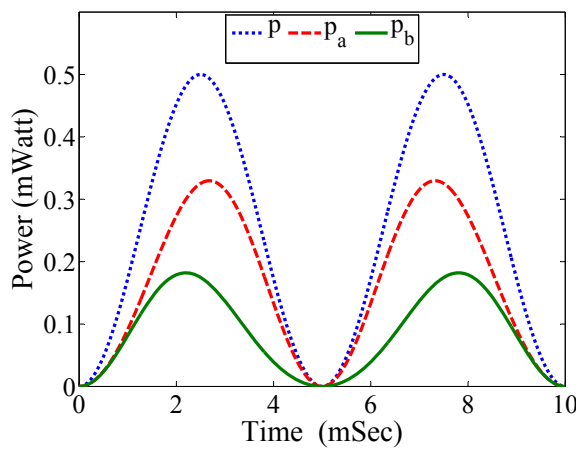

(a)

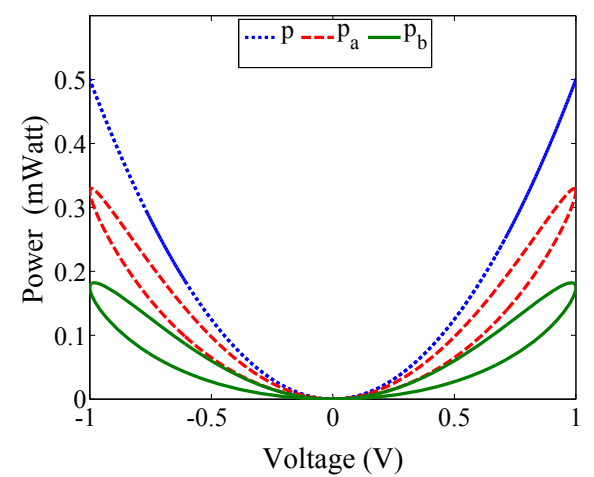

(b)

Fig. 6. Plot of power consumption in $R_{a}$ and $R_{b}$ for $\alpha=-1$.

\section{Power of Memristor-Based Relaxation Oscillator}

The oscillator circuit oscillates for three cases only which are $\alpha=0, \infty$ and -1 and it wouldn't oscillate for $\alpha=1$ since the ratio voltage to comparators is always constant as discussed in [15]. The output of these oscillators is either $V_{\mathrm{oh}}$ or $V_{\mathrm{ol}}$ which are the maximum and minimum output voltages respectively. So, the input to the memristive is either $V_{\mathrm{oh}}$ or $V_{\mathrm{ol}}$ for single gate oscillator but in the case of a two gate oscillator, the input to the memristive circuit is either $V_{\mathrm{oh}}-V_{\mathrm{ol}}$ or $V_{\mathrm{ol}}-V_{\mathrm{oh}}$. The power consumption in the oscillator is divided into two regions; the first when the output is $V_{\mathrm{oh}}$, and the other, when the output is $V_{\mathrm{ol}}$. In this section, the power dissipation in the single gate oscillator is mathematically calculated. Moreover, in the case of a two gate oscillator, by replacing $V_{\mathrm{oh}}$ and $V_{\mathrm{ol}}$ with $V_{\mathrm{oh}}-V_{\mathrm{ol}}$ and $V_{\mathrm{ol}}-V_{\mathrm{oh}}$ respectively, similar expressions can be found.

The general equation of power dissipation oscillators is given by

$$
p(t)= \begin{cases}\frac{V_{\mathrm{ol}}^{2}}{\sqrt{\left(R_{\mathrm{ind}}^{2}+(\alpha+1) R_{\mathrm{inb}}\right)^{2}-2(\alpha+1) k_{\mathrm{b}}^{\prime} V_{\mathrm{ol}} \tau}} & 0<\tau<T_{l}, \\ \frac{V_{\mathrm{oh}}^{2}}{\sqrt{\left(R_{\mathrm{ind}}^{2}+(\alpha+1) R_{\mathrm{inb}}\right)^{2}-2(\alpha+1) k_{\mathrm{b}}^{\prime} V_{\mathrm{oh}}\left(\tau-T_{1}\right)}} & T_{1}<\tau<T,\end{cases}
$$

where $T_{1}$ is the duration of $V_{\mathrm{oh}}, T$ is the period of the oscillator, and $\tau=\bmod \left(t-t_{\text {trans }}, T\right)$ where $t_{\text {trans }}$ is the transition time.

Consequently, the power dissipation in the three aforementioned special cases can be studied as follows:

1. $\alpha=0$.

The transient power dissipation by $R_{a}$ and the total power consumption are given by

$$
\begin{array}{r}
p_{\mathrm{a}}(t)= \begin{cases}\frac{V_{\mathrm{ol}}^{2} R_{\mathrm{a}}}{\left(R_{\mathrm{a}}+R_{\mathrm{bn}}\right)^{2}-2 k_{\mathrm{b}}^{\prime} V_{\mathrm{ol}} \tau} & 0<\tau<T_{1}, \\
\frac{V_{\mathrm{oh}}^{2} R_{\mathrm{a}}}{\left(R_{\mathrm{a}}+R_{\mathrm{bp}}\right)^{2}-2 k_{\mathrm{b}}^{\prime} V_{\mathrm{oh}}\left(\tau-T_{\mathrm{l}}\right)} & T_{1}<\tau<T,\end{cases} \\
p(t)= \begin{cases}\frac{V_{\mathrm{ol}}^{2}}{\sqrt{\left(R_{\mathrm{a}}+R_{\mathrm{bn}}\right)^{2}-2 k_{\mathrm{b}}^{\prime} V_{\mathrm{ol}} \tau}} & 0<\tau<T_{1}, \\
\frac{V_{\mathrm{oh}}^{2}}{\sqrt{\left(R_{\mathrm{a}}+R_{\mathrm{bp}}\right)^{2}-2 k_{\mathrm{b}}^{\prime} V_{\mathrm{oh}}\left(\tau-T_{\mathrm{l}}\right)}} & T_{1}<\tau<T,\end{cases}
\end{array}
$$

where $R_{\mathrm{bn}}$ and $R_{\mathrm{bp}}$ are the maximum and minimum achievable memristances $R_{\mathrm{b}}$ are $R_{\mathrm{a}} V_{\mathrm{n}} /\left(V_{\mathrm{ol}}-V_{\mathrm{n}}\right)$ and $R_{\mathrm{a}} V_{\mathrm{p}} /\left(V_{\mathrm{oh}}-V_{\mathrm{p}}\right)[13]$. Clearly, the power consumption is a function of $R_{\mathrm{a}}, R_{\mathrm{bn}}$ and $R_{\mathrm{bp}}$ and $R_{\mathrm{a}}$ should be enclosed between $R_{\mathrm{on}}\left(V_{\mathrm{ol}}-V_{\mathrm{n}}\right) / V_{\mathrm{n}}$ and $R_{\mathrm{off}}\left(V_{\mathrm{oh}}-V_{\mathrm{p}}\right) / V_{\mathrm{p}}$ for sustained oscillation, as discussed in [13]. Thus, the total maximum and minimum power consumption are given by

$$
\begin{aligned}
& p_{\text {max }}=\frac{V_{\mathrm{ol}}^{2}}{R_{\mathrm{a}}+R_{\mathrm{bn}}}=\frac{V_{\mathrm{ol}} V_{\mathrm{n}}}{R_{\mathrm{on}}}, \\
& p_{\text {min }}=\frac{V_{\mathrm{ol}}^{2}}{R_{\mathrm{a}}+R_{\mathrm{bp}}}=\frac{V_{\mathrm{oh}} V_{\mathrm{p}}}{R_{\mathrm{off}}},
\end{aligned}
$$

respectively.

The PSPICE simulation of the power consumption across the memristor, the resistor and the sum are displayed in Fig. 7 showing perfect matching. PSPICE simulations are performed using the spice model proposed in [9]. This figure is plotted for single gate oscillator at the following setup, $k_{\mathrm{b}}^{\prime}, V_{\mathrm{p}}, V_{\mathrm{n}}, V_{\mathrm{oh}}, V_{\mathrm{ol}}$ and $R_{\mathrm{a}}$ are $-379 \mathrm{M}^{2} / \mathrm{C}, 0.75 \mathrm{~V},-0.5 \mathrm{~V}, 1 \mathrm{~V},-1 \mathrm{~V}, 3 \mathrm{k} \Omega$. The memristance changes from $R_{\mathrm{mn}}=3 \mathrm{k} \Omega$ to $R_{\mathrm{mp}}=9 \mathrm{k} \Omega$ so the oscillator oscillates with frequency $3.51 \mathrm{~Hz}$.

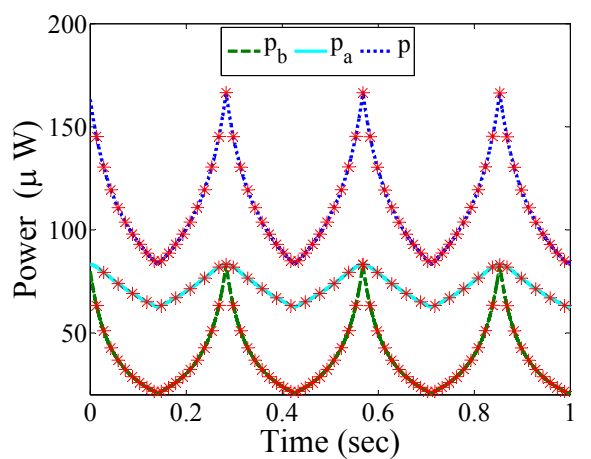

Fig. 7. PSPICE (solid line) and numerical simulation (stars) power consumption in single gate memristor-based oscillator for $\alpha=0$. 
2. $\alpha= \pm \infty$.

The transient power dissipation by $R_{\mathrm{b}}$ and the total power consumption are given by

$$
\begin{gathered}
p_{\mathrm{b}}(t)= \begin{cases}\frac{V_{\mathrm{ol}}^{2} R_{\mathrm{b}}}{\left(R_{\mathrm{b}}+R_{\mathrm{an}}\right)^{2}-2 k_{\mathrm{a}}^{\prime} V_{\mathrm{ol}} \tau} & 0<\tau<T_{\mathrm{l}}, \\
\frac{V_{\mathrm{oh}}^{2} R_{\mathrm{b}}}{\left(R_{\mathrm{b}}+R_{\mathrm{ap}}\right)^{2}-2 k_{\mathrm{a}}^{\prime} V_{\mathrm{oh}}\left(\tau-T_{\mathrm{l}}\right)} & T_{1}<\tau<T,\end{cases} \\
p(t)= \begin{cases}\frac{V_{\mathrm{ol}}^{2}}{\sqrt{\left(R_{\mathrm{b}}+R_{\mathrm{an}}\right)^{2}-2 k_{\mathrm{a}}^{\prime} V_{\mathrm{ol}} \tau}} & 0<\tau<T_{\mathrm{l}}, \\
\frac{V_{\mathrm{oh}}^{2}}{\sqrt{\left(R_{\mathrm{b}}+R_{\mathrm{ap}}\right)^{2}-2 k_{\mathrm{a}}^{\prime} V_{\mathrm{oh}}\left(\tau-T_{\mathrm{l}}\right)}} & T_{1}<\tau<T,\end{cases}
\end{gathered}
$$

where $R_{\text {an }}$ and $R_{\text {ap }}$ are the maximum and minimum achievable memristance $R_{\mathrm{a}}$ are $R_{\mathrm{b}}\left(V_{\mathrm{ol}}-V_{\mathrm{n}}\right) / V_{\mathrm{n}}$ and $R_{\mathrm{b}}\left(V_{\mathrm{oh}}-V_{\mathrm{p}}\right) / V_{\mathrm{p}}$ respectively [13]. Clearly, the power consumption is a function of $R_{\mathrm{b}}, R_{\mathrm{an}}$ and $R_{\mathrm{ap}}$ and $R_{\mathrm{b}}$ should be enclosed between $R_{\mathrm{on}} V_{\mathrm{n}} /\left(V_{\mathrm{ol}}-V_{\mathrm{n}}\right)$ and $R_{\mathrm{off}} V_{\mathrm{p}}\left(V_{\mathrm{oh}}-V_{\mathrm{p}}\right)$ for sustained oscillation, as discussed in [13]. Thus, the total maximum and minimum power consumption are given by

$$
\begin{aligned}
& p_{\max }=\frac{V_{\mathrm{ol}}^{2}}{R_{\mathrm{b}}+R_{\mathrm{an}}}=\frac{V_{\mathrm{ol}}\left(V_{\mathrm{ol}}-V_{\mathrm{n}}\right)}{R_{\mathrm{on}}}, \\
& p_{\min }=\frac{V_{\mathrm{ol}}^{2}}{R_{\mathrm{b}}+R_{\mathrm{ap}}}=\frac{V_{\mathrm{oh}}\left(V_{\mathrm{oh}}-V_{\mathrm{p}}\right)}{R_{\mathrm{off}}},
\end{aligned}
$$

respectively.

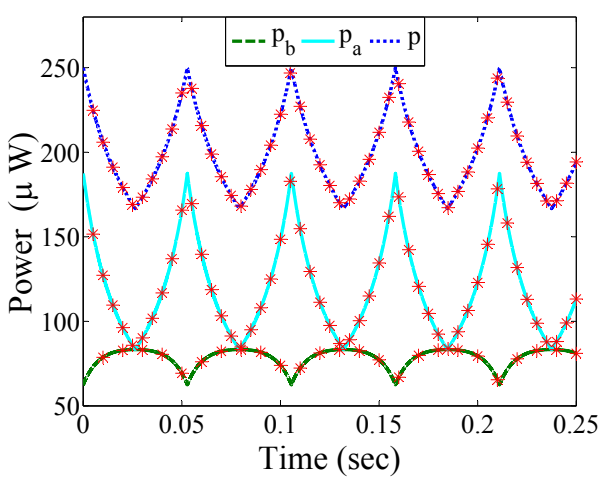

Fig. 8. PSPICE (solid line) and numerical simulation (stars) of power consumption for $\alpha=\infty$.

Figure 8 shows the transient PSPICE simulation of the power consumption across the memristor, the resistor and the sum showing good matching with the calculated expressions. This figure is plotted for single gate oscillator at the following setup $k_{\mathrm{a}}^{\prime}, V_{\mathrm{p}}, V_{\mathrm{n}}, V_{\mathrm{oh}}, V_{\mathrm{ol}}$ and $R_{\mathrm{b}}$ are $-379 \mathrm{M} \Omega^{2} / \mathrm{C}, 0.5 \mathrm{~V},-0.75 \mathrm{~V}, 1 \mathrm{~V},-1 \mathrm{~V}, 3 \mathrm{k} \Omega$. The memristance changes from $R_{\mathrm{an}}=1 \mathrm{k} \Omega$ to $R_{\mathrm{ap}}=3 \mathrm{k} \Omega$ and the oscillation frequency is $18.95 \mathrm{~Hz}$.

3. $\alpha=-1$.

The transient power dissipation by $R_{\mathrm{a}}, R_{\mathrm{b}}$ and total power are given by

$$
p_{\mathrm{a}}(t)= \begin{cases}\frac{V_{\mathrm{ol}}^{2}\left(R_{\mathrm{an}}\left(R_{\mathrm{ina}}+R_{\mathrm{inb}}\right)-k_{\mathrm{a}}^{\prime} V_{\mathrm{ol}} \tau\right)}{\left(R_{\mathrm{ina}}+R_{\mathrm{inb}}\right)^{3}} & 0<\tau<T_{1}, \\ \frac{V_{\mathrm{oh}}^{2}\left(R_{\mathrm{ap}}\left(R_{\mathrm{ina}}+R_{\mathrm{inb}}\right)-k_{\mathrm{a}}^{\prime} V_{\mathrm{oh}}\left(\tau-T_{1}\right)\right)}{\left(R_{\mathrm{ina}}+R_{\mathrm{inb}}\right)^{3}} & T_{1}<\tau<T,\end{cases}
$$

$$
\begin{gathered}
p_{\mathrm{b}}(t)= \begin{cases}\frac{V_{\mathrm{ol}}^{2}\left(R_{\mathrm{bn}}\left(R_{\mathrm{ina}}+R_{\mathrm{inb}}\right)-k_{\mathrm{b}}^{\prime} V_{\mathrm{ol}} \tau\right)}{\left.R_{\text {ina }}+R_{\text {inb }}\right)^{3}} & 0<\tau<T_{1}, \\
\frac{V_{\mathrm{oh}}^{2}\left(R_{\mathrm{bp}}\left(R_{\mathrm{ina}}+R_{\text {inb }}\right)-k_{\mathrm{b}}^{\prime} V_{\mathrm{oh}}\left(\tau-T_{1}\right)\right)}{\left(R_{\text {ina }}+R_{\text {inb }}\right)^{3}} & T_{1}<\tau<T,\end{cases} \\
p(t)= \begin{cases}\frac{V_{\mathrm{ol}}^{2}}{R_{\text {ina }}+R_{\text {inb }}} & 0<\tau<T_{1}, \\
\frac{V_{\mathrm{oh}}^{2}}{R_{\text {ina }}+R_{\text {inb }}} & T_{1}<\tau<T .\end{cases}
\end{gathered}
$$

where, [14],

$$
\begin{gathered}
R_{\mathrm{an}}=\left(R_{\text {ina }}+R_{\text {inb }}\right)\left(V_{\mathrm{ol}}-V_{\mathrm{n}}\right) / V_{\mathrm{ol}}, \\
R_{\text {ap }}=\left(R_{\text {ina }}+R_{\text {inb }}\right)\left(V_{\mathrm{oh}}-V_{\mathrm{p}}\right) / V_{\mathrm{oh}}, \\
R_{\mathrm{bn}}=\left(R_{\mathrm{ina}}+R_{\mathrm{inb}}\right) V_{\mathrm{n}} / V_{\mathrm{ol}}, \\
R_{\mathrm{bp}}=\left(R_{\text {ina }}+R_{\text {inb }}\right) V_{\mathrm{p}} / V_{\mathrm{oh}} .
\end{gathered}
$$

It is obvious that the power dissipation in this case is a function of the sum of the memristances, $R_{\mathrm{S}}=R_{\mathrm{a}}+R_{\mathrm{b}}$, which is always constant. But, to obtain an oscillation, $R_{\mathrm{S}}$ should be between a certain range, given in [14]. Using the minimum and maximum $R_{\mathrm{s}}$, the total maximum and minimum power consumption are given by

$$
\begin{aligned}
& p_{\text {max }}=\frac{V_{\mathrm{ol}}\left(V_{\mathrm{ol}}-V_{\mathrm{n}}\right)}{R_{\mathrm{on}}}, \\
& p_{\text {min }}=\frac{V_{\mathrm{oh}}\left(V_{\mathrm{oh}}-V_{\mathrm{p}}\right)}{R_{\mathrm{off}}},
\end{aligned}
$$

respectively.

Figure 9 shows good matching between the calculated expressions and PSPICE simulations, where $k_{\mathrm{a}}^{\prime}, k_{\mathrm{b}}^{\prime}$, $V_{\mathrm{p}}, V_{\mathrm{n}}, V_{\mathrm{oh}}, V_{\mathrm{ol}}$ and $R_{\mathrm{b}}$ are $379 \mathrm{M} \Omega^{2} / \mathrm{C},-379 \mathrm{M} \Omega^{2} / \mathrm{C}$, $0.75 \mathrm{~V}, 0.75 \mathrm{~V}, 1 \mathrm{~V},-1 \mathrm{~V}, 3 \mathrm{k} \Omega$. The memristance changes from $R_{\mathrm{an}}=1 \mathrm{k} \Omega$ to $R_{\mathrm{ap}}=0.5 \mathrm{k} \Omega$ and $R_{\mathrm{bn}}=1 \mathrm{k} \Omega$ to $R_{\mathrm{bp}}=1.5 \mathrm{k} \Omega$ with oscillation frequency of $189.5 \mathrm{~Hz}$.

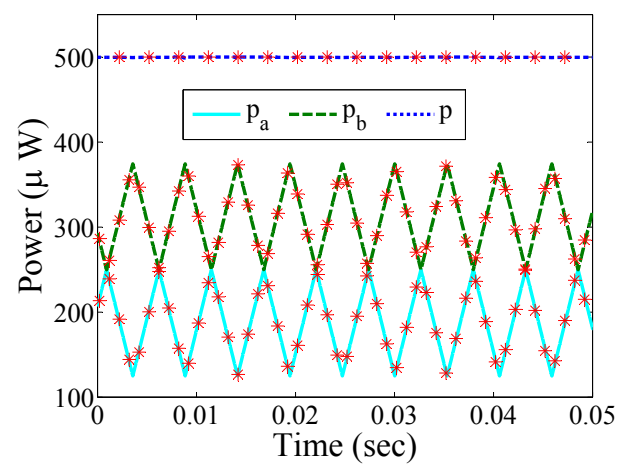

Fig. 9. PSPICE (solid line) and numerical simulation (stars) power consumption for $\alpha=-1$.

It is clear that, the maximum power consumption in all cases is linearly proportional to $\left|V_{\mathrm{n}}\right|$ and inversely proportional to $R_{\mathrm{on}}$, however, the minimum power consumption is linearly proportional to $V_{\mathrm{p}}$ and inversely proportional to $R_{\text {off. Also, the maximum and minimum power consumptions }}$ happen at maximum and minimum oscillation frequencies. 


\section{Conclusion}

Through this paper, we introduced mathematical expressions for the power dissipation of memristor-based relaxation oscillators. Maximum and minimum power consumption were introduced for different cases which are function of the biasing parameters of the oscillator and maximum as well as the minimum achievable memristances. It is clear from this discussion that the instantaneous power consumption has even-symmetric pinched hysteresis and the power dissipation is a function of the oscillation frequency. Moreover, The power consumption of three topologies are calculated and verified using PSPICE simulations. The introduced analyses can be generalized for different memristor models and for other memristor-based oscillators.

\section{References}

[1] CHUA, L. Memristor - the missing circuit element. IEEE Transactions on Circuit Theory, 1971, vol. 18, no. 5, p. 507-519. DOI: 10.1109/TCT.1971.1083337

[2] STRUKOV, D., SNIDER, G., STEWART, D., WILliAMS, R. The missing memristor found. Nature, 2008, vol. 453, p. 80-83. DOI:10.1038/nature06932

[3] KOZMA, R., PINO, R. E., PAZIENZA, G. E. Advances in Neuromorphic Nemristor Science and Applications, vol. 4. Springer, 2012. DOI:10.1007/978-94-007-4491-2

[4] RADWAN, A. G., FOUDA, M. E. On the Mathematical Modeling of Memristor, Memcapacitor, and Meminductor. Springer, 2015. DOI: 10.1007/978-3-319-17491-4

[5] KIM, H., SAH, M., YANG, C., CHO, S., CHUA, L. Memristor emulator for memristor circuit applications. IEEE Transactions on Circuits and Systems I: Regular Papers, 2012, vol. 59, no. 10, p. 2422-2431. DOI:10.1109/TCSI.2012.2188957

[6] ELWAKIL, A., FOUDA, M., RADWAN, A. A simple model of double-loop hysteresis behavior in memristive elements. IEEE Transactions on Circuits and Systems II: Express Briefs, 2013, vol. 60 , no. 8, p. 487-491. DOI:10.1109/TCSII.2013.2268376

[7] HUSSEIN, A., FOUDA, M. A simple MOS realization of curren controlled memristor emulator. In 25th IEEE International Conference on Microelectronics (ICM). Beirut (Lebanon), 2013, p. 1-4. DOI:10.1109/ICM.2013.6734969

[8] FOUDA, M., RADWAN, A. simple floating voltage-controlled memductor emulator for analog applications. Radioengineering, 2014 vol. 23 , no. 3 ., p. $944-948$.

[9] BIOLEK, Z., BIOLEK, D., BIOLKOVA, V. SPICE model of memristor with nonlinear dopant drift. Radioengineering, 2009, vol. 18, no. 2 , p. $210-214$

[10] PERSHIN, Y. V., DI VENTRA, M. SPICE model of memristive devices with threshold. Radioengineering, 2013, vol. 22, no. 2 ., p. 485-489.

[11] BIOLEK, D., DI VENTRA, M., PERSHIN, Y. Reliable SPICE simulations of memristors, memcapacitors and meminductors. Radioengineering, 2013, vol. 22 , no. 4, p. 945-968.
[12] TALUKDAR, A., RADWAN, A., SALAMA, K. Generalized model for memristor-based Wien family oscillators. Microelectronics Journal, 2011, vol. 42, no. 9, p. 1032-1038. DOI:10.1016/j.mejo.2011.07.001

[13] FOUDA, M., RADWAN, A. Memristor-based voltage-controlled relaxation oscillators. International Journal of Circuit Theory and Applications, 2014, vol. 42, no. 10, p. 1092-1102. DOI:10.1002/cta.1907

[14] ZIDAN, M., OMRAN, H., SMITH, C., SYED, A., RADWAN, A. SALAMA, K. A family of memristor-based reactance-less oscillators. International Journal of Circuit Theory and Applications, 2014, vol. 42, no. 11, p. 1103-1122. DOI:10.1002/cta.1908

[15] FOUDA, M. E., KHATIB, M., MOSAD, A., RADWAN, A. Generalized analysis of symmetric and asymmetric memristive twogate relaxation oscillators. IEEE Transactions on Circuits and Systems I: Regular Papers, 2013, vol. 60, no. 10, p. 2701-2708. DOI:10.1109/TCSI.2013.2249172

\section{About the Authors...}

Mohammed FOUDA received the B.Eng. degree (honors), in Electronics and Communications Engineering in 2011 from Faculty of Engineering, Cairo University, Cairo, Egypt. Also, Fouda received his MSc degree in Engineering Mathematics in 2014 from the same university. He is an Assistant Lecturer at Engineering Mathematics and Physics Department, Cairo University, Egypt. He authored and coauthored more than 23 journal and conference papers, and one Springer book. Fouda won the best paper award in ICM 2013 in Lebanon. His research interests include Memelements based circuits, and analog circuits.

Ahmed RADWAN received the B.S. degree (honors) in Electronics Engineering, and the M.S. and Ph.D. degrees from Cairo University, Cairo, Egypt, in 1997, 2002, and 2006 respectively. He is an Associate Professor in the Engineering Mathematics Department, Cairo University, Egypt, and Director of Nanoelectronics Integrated Systems Center (NISC), Nile University, Egypt. He is Former Director of the Technical Center for Career Development (TCCD), Cairo University, Egypt. He was invited as a visiting professor with the Computational Electromagnetics Lab, ECE, McMaster University, Canada. From 2010 to 2012, he was selected to be one to form the pioneer research group in the King Abdullah University of Science and Technology (KAUST), Saudi Arabia. He is a senior member of IEEE, received the State achievements award for research in the Mathematical Sciences in 2012, received the Cairo University achievements award for research in the Engineering Sciences in 2013, Best researcher awards Nile University 2015, and won the Physical Sciences award in the 2013 by Misr El-Khair Institution. His main research interests are in the fields of nonlinear circuit analysis, chaotic systems, fractional order systems, and memristor-based circuits. He is the coauthor of more than 140 international papers, many international books, and six US Patents. 